\title{
Checklist Of The Aquatic Macrophyte Flora Abundence In Lower Manair Dam At Karimnagar Dt. Andhra Pradesh, India.
}

\author{
*Rama Rao. K., **Ramakrishna. $\mathrm{N}$ and $* * *$ Amravati. D \\ *Department of Zoology, Govt. Degree College, Jammikunta, Satavahana University, Andhra Pradesh \\ ** PGT in Biology, Sainik School, Korukonda, Vizianagaram, Andhra Pradesh \\ *** Biology, Chitanya College of Education, Bonagi, Parawada, Visakhapatnam. A.P. India.
}

\begin{abstract}
The present investigation concerns with the aquatic macrophyte diversity and its role in understanding the reservoir ecosystem dynamics and species composition of aquatic macrophytes distribution in ten villages around Lower Manair Dam (LMD) were studied during 2013-2014. Forty eight different species of aquatic macrophytes were recorded in 04 classes, 20 orders and 26 families, which include five free floating macrophytes, ten submerged, and thirty three emergent species of weds were recorded. The percentage composition of class, order and families were calculated during this period. The free floating species Eichornia crassipes occurs throughout the year, while the submerged species Vallisneria spiralis, Ceratophyllum demursum, Hydrilla verticillata occur throughout the year. In the rooted floating category Ipomoea aquatica occurs throughout the year. The common name, habitat and IUCN (2013.2) status of the macrophytes has also been noted in the present paper.
\end{abstract}

Key words: Macrophytes, Free floating, Submerged, Emergent, LMD.

\section{Introduction}

Lower Manair Dam (LMD) is situated in Karimnagar District of Telangana region (Fig. 1). This is a large new impoundment of Godavari basin with medium productive potential. The Lower Manair Dam is situated at Kakatiya Canal about $146.00 \mathrm{~km}$ to $234 \mathrm{kms}$ and Distributaries D 84 to D 94 and DBM 1 to DBM2. LMD water goes up to 2, 62,326 acs, catchment area of river 6,475 sq.m. Reservoir full level is $280.416 \mathrm{mt}$. Full capacity of reservoir $0.68 \mathrm{TM}$ Cusecs and water spread area is 81.024 sq. kmt. The Lower Manair Dam harbors a variety of macrophytes in different sub-stratus. Macrophytes serve as a link between the sediment, water, and atmosphere in reservoirs, lakes, and rivers. The most notable function that plants serve is as primary producers. However, macrophytes are also involved in ecosystem processes such as biomineralization, transpiration, sedimentation, elemental cycling, materials transformation, and release of biogenic trace gases into the atmosphere (Carpenter and Lodge, 1986). Recent studies also suggest that macrophytes play a central role in shallow reservoirs which can have two possible stable equilibrium: a clear-water state that is dominated by aquatic macrophytes and a turbid-water state that is dominated by phytoplankton (Jeppesen et al., 1998). Aquatic weeds referred to as Macrophytes constitute an important component of aquatic ecosystem. Their diversity and biomass influence primary productivity and complexities of tropic states (Kumar and Singh, 1987). Since the wetland is considered as a transitional area between land and water, Smith (1980) aptly described it as a half-way world between terrestrial and aquatic ecosystems. It is largely dominated by water and has special type of flora and fauna, which usually undergo time scheduled characteristic changes from hydric to mesic types. Wetlands are well known for high diversity in class, composition and four broad categories of functions viz. physical, hydrological, chemical, biological and socioeconomic (Williams,1990).

The study of submerged and floating vegetation is of great importance as far as food supply to fish species is concerned. The menace of aquatic weeds can be overcome by their utilization in a number of ways. Lower Manair Dam had been rich fish productivity and in multi dimensional utilization of aquatic weeds Rama Rao. K (2014 a. b) reported 53 ornamental species and 58 larvivorous fishes in this reservoir out of these, 13 species were abundant, 13 species were common, 19 species were moderate and seven species were rare. Aquatic plants constitute an integral component of an aquatic ecosystem. They may serve as good source of food to the mankind, a palatable feed to the water birds and animals thus forming a base for aquatic wildlife conservation practices. They also serve as a potential source of energy (Majid, 1986). Macrophytes of different water bodies in India are studied by researchers such as Wetzel (1975), Majid (1986), Meshram (2003), Ambasht (2005) and Raut and Pejawer, (2005) and many more.

\section{Material And Methods}

The studies were carried out from April 2013 March 2014 during different seasons at four different sites from the reservoir catchment area. Macrophytes were collected during three different seasons such as monsoon, winter and summer season by field visit to the spots. The survey was conducted to collect the information 
regarding floating, emergent, and submerged vegetation. The macrophytes were collected by hand picking, and also using nets and then brought to laboratory preserved in $10 \%$ formalin and identified using standard available literature (Cook, 1996). The identification of aquatic plants was done with the help of standard books and monographs like, Singh and Karthikeyan (2000 and 2001), Biswas and Calder (1953). The data collected was used to analyses and diversity indices were calculated.

\section{Results And Discussion}

The results of the present study revealed that the occurrence of forty eight weed species belong to twenty orders, 33 families and four classes. List of hydrophytes including their class, order, family, genus, species and common name were recorded in the present investigation was given in Table 1, 2, 3. The listed species under free floating are Azolla filiculoides, Eichornia crassipes (Mart.) Solms, Lemna perpusilla Torrey, Ottelia alismoides (L.) Pers., and Pistia stratiotes L. (Table: 1), the Submerged species are Aponogeton natans L.f, Chara globularis J. L.T, Ceratophyllum demersum L., Hydrilla verticillata (L. f.) Royle., Ipomoea aquatica Forsk (Rooted)., Ipomoea carnea jacq, Marsilea quadrifolia, Najas minor L., Potamogeton pectinata L., Vallisneria spiralis L. . (Table: 2), The emergent weeds are Alternanthera sessilis (L.)R. Br. Ex, Alternanthera philoxeroides, Ammannia baccifera L., Aeschynomene indica L., Bacopa monnieri (L.) Wettestin, Coix aquatica Roxb., Commelina benghalensis L., Commelina hasskarlii C. Comm. Cyrt., Cyperus rotundus L., Cyperus difformis L., Cynodon dactylon (L.) Pers., Cyathocline purpurea (Buch-Ham. ex D. Don) Oktze., Chrozophora rottleri (Geisel.) A. Juss. ex. Spr., Dopatrium junceum (Roxb.) Buch- Hum ex Benth., Eclipta alba, Eleocharis geniculata (L.) R\&S., Eleocharis capitata R. Br., Eriocaulon cinereum R.Br., Echinochloa colona (L.) Link. Fimbristylis miliacea, Gomphrena celosioides Mart., Glinus lotoides L., Gnaphalium pulvinatum Del., Grangea, maderaspatana., Heliotropium supinum L. Limnophila sessiliflora L. Polygonum glabrum Willd. Phyla nodiflora (L.) Greene. Rotala serpyllifolia (Roth.) Bremeck., Sesbania bispinosa (Jacq.) w. t. wight., Sopubia delphinifolia (L.) G. Don, Sphaeranthus indicus L., Typha angustata Bory and Chaub.

Thomaz.et al., (1999, 2001) found aquatic macrophytes of Itaipu Reservoir at Brazil and a similar study was conducted by Bartodziej, and Ludlow (1997) performed to aquatic vegetation monitoring by natural resource agencies in the United States. Chambers et al., (2010) were classified world checklist of macrophyte species. Patil et al., (2012) explained the hydrophytes and amphibious plants occurred in Panchganga River in vicinity of Ichalkaranji city district Kolhapur, Maharashta.

The number of classes, orders and families under three types of weeds were shown in Table 4. Fig: 1. the free floating and emergent weeds are contributed each two class and submerged weeds contributed highest for four classes in LMD. Recorded aquatic weeds were under twenty orders, the emergent weeds occupied highest 13 species, followed by submerged weeds (06) and free floating weeds (04). In 26 families the emergent weeds occupied highest are 15, followed by submerged weeds (06) and free floating weeds (05). Emergent weeds are dominant with 33 species which contributes $68.75 \%$ of the total species followed by submerged weeds with $10(20.83 \%)$ and free floating weeds with $05(10.42 \%)$.

The number and percentage composition of free floating, submerged and emergent weeds were under four classes Liliopsida was dominant with four species which contributed to $80 \%$ of the total five species and followed by Polypodiopsida was one species which contributed to $20 \%$ of the total free floating aquatic weeds. Recorded submerged weeds out of ten species, Liliopsida contributed five species (50\%), followed by Magnoliopsida three species (30\%), Polypodiopsida and Charophyceae each with one species (10\%). Recorded emergent weeds out of 33 species, Magnoliopsida contributed with 21 (63.64\%) species, followed by Liliopsida with 12 species $(36.37 \%)$.

The number and percentage composition of free floating, submerged and emergent weeds were under twenty orders, Arales was dominant with two species which contributed to $40 \%$ of the total five species of free floating weeds and followed by Commelinales, Hydrocharitales and Salviniales each with one species (20\%). Recorded submerged weeds out of ten species Hydrocharitales contributed three species (30\%) followed by Najadales and Solanales each with two species (20\%) and Salviniales, Charales and Nymphaeales each with one species (10\%). Recorded emergent weeds out of 33 species, Asterales was dominant with five species which contributed 15.15\%, followed by Caryophyllales, Poales and Scrophulariales each with four species (12.12\%), Commelinales, Fabales and Myrtales each with two species (6.06\%) and Boraginales, Lamiales, Malpighiales, Polygonales and Typhales which contributed to each one species (3.03\%). Recorded 48 species of aquatic weeds are Asterales and Cyperales were dominant with each five species which contributed to $10.42 \%$ and followed by Hydrocharitales, Caryophyllales, Poales and Scrophulariales were each with four species (8.33\%), Commelinales was dominant with three species (6.25\%), Arales, Salviniales, Najadales, Solanales, Fabales, and Myrtales each with two species (4.17\%), Charales, Nymphaeales, Boraginales, Lamiales, Malpighiales, Polygonales and Typhales contributed each one species $(2.08 \%)$.

The number and percentage composition of free floating, submerged and emergent weeds were under 26 families, Araceae, Hydrocharitaceae, Lemnaceae, Pontederiaceae and Salviniaceae each with one species 
which contributed to $20 \%$ of the total five species of free floating weeds. Out of recorded ten species of submerged weeds are Hydrocharitaceae was dominant with three species (30\%) followed by Convolvulaceae with two species (20\%), Aponogetonaceae, Characeae, Ceratophyllaceae, Marsileaceae and Potamogetonaceae contributed to each with one (10\%) species. Out of recorded 33 species of emergent weeds Asteraceae and Cyperaceae were dominant with five $(15.15 \%)$ species followed by Scrophulariaceae with four $(12.12 \%)$ species, Amaranthaceae and Poaceae each with three (9.09\%) species, Commelinaceae, Fabaceae and Lythraceae each with two (6.06\%) species, Boraginaceae, Eriocaulaceae, Euphorbiaceae, Molluginaceae, Polygonaceae, Verbenaceae and Typhaceae each with one (3.03\%). Recorded 48 species of aquatic weeds are under 26 families; Asteraceae and Cyperaceae were dominated each with five species, which contributed to $10.42 \%$ of the total species. Hydrocharitaceae and Scrophulariaceae contributed each with four $(8.33 \%)$ species. Amaranthaceae and Poaceae contributed each with three (6.25\%) species. Convolvulaceae, Commelinaceae, Fabaceae and Lythraceae Scrophulariaceae contributed each with two (4.16\%) species. Araceae, Lemnaceae, Pontederiaceae, Salviniaceae, Aponogetonaceae, Characeae, Ceratophyllaceae, Marsileaceae, Potamogetonaceae, Boraginaceae, Eriocaulaceae, Euphorbiaceae, Molluginaceae, Polygonaceae, Verbenaceae and Typhaceae contributed each with one (2.08\%) species.

An assessment of macrophyte biodiversity of a freshwater reservoir of Bhadrawati Tehsil in Chandrapur district, by Shashikant R.Sitre (2013). Gokhele, et al (2011) conducted a survey on flora of wet coastal and associated ecosystem to Maharastra. The similar study was conducted by Kayode, J. and Ogunleye, O.T. (2008) who classified the macrophytes checklist and status of plant species used as spices in Kaduna state of Nigeria.

A total of 48 species of hydrophytes belonging to 4 classes, 20 orders and 26 families were studied. According to IUCN (2013.2) of these 35 species of aquatic weeds are least concerned, 12 are not evaluate (NE), 1 species is vulnerable (VU). In the free floating weeds, three species are least concerned (6.25\%), two (4.17\%) species are not evaluated. In the submerged weeds, eight species (16.67\%) are least concerned and two $(4.17 \%)$ species are not evaluated. In the emergent weeds 24 species (50\%) are least concerned, eight (16.67\%) are not evaluated; one $(2.03 \%)$ is vulnerable.

James et al (2006) surveyed aquatic plants in 38 reservoirs throughout Arizona from 2004 to develop an inventory of species and to determine species distribution and composition patterns. They identified 12 submersed, 3 floating, and 18 emergent aquatic plants to species level; some samples were only identified to genera, and none of the filamentous algae were identified to either genera or species. Adesina, (2007) studied an assessment of aquatic vegetation of Jebba Lake, Nigeria. Olsen, S (1950) and Spence, D.H.N. (1964) represented aquatic plants and hydrospheric factor and the macrophytes vegetation of lochs, swamps and associated fens. Ambasht, R. S. (2005), Billore, D. K. and Vyas, I. N. (1981) studied the macrophytes limnology, distribution and production of macrophytes in the Indian subcontinent. Kiran, B.R. et al., (2006) Krull, J.N. 1970, Madsen, J.D. and P.A. Chambers (2001) studied aquatic macrophytes in fish culture ponds, aquatic plant-invertebrate associations and the Interactions between water movement, sediment dynamics and submersed macrophytes.

\section{Conclusion}

A number of macrophyte species are available in Lower Manair Dam at monsoon period and post monsoon season. Free floating and submerged species are more dominant in all seasons. Emergent weed species are dominant in the winter season. Presently, the fresh water aquatic weeds are directly or indirectly more helpful for food and shelter to reservoir fishery at Lower Manair Dam.

\section{Acknowledgments}

The authors would like to thank University Grants Commission for rendering financial assistance and Commissioner of Collegiate Education Andhra Pradesh, Govt. Degree College, Jammikunta for providing necessary facilities.

\section{References}

[1]. Adesina, G. O., Akinyemiju, O.A. and Olaleye, V.F. (2007) Assessment of aquatic vegetation of Jebba Lake, Nigeria. African Journal of Ecology 45:365-373.

[2]. Ambasht, R. S. (2005). Macrophytes limnology in the Indian subcontinent. Ukaaz Publication, Hyderabad: 58 - 174.

[3]. Bartodziej, W. and J. Ludlow. (1997). Aquatic vegetation monitoring by natural resource agencies in the United States. Lake Reserv. Manage. 13:109-117.

[4]. Billore, D. K. and Vyas, I. N. (1981) Distribution and production of macrophytes in pichhola lake, Udaipur. Dnt J Ecol Env-sci 7:45-54.

[5]. Biswas, K. and Calder, L. C. (1984) Handbook of common water and marsh plants of India and Burma, xvii 216, Bishensingh Mahendra palsingh [Dehradun].

[6]. Carpenter, S. R., Lodge, D. M. (1986) Effects of submersed macrophytes on ecosystem processes. Aquatic Bot 26: 341-370.

[7]. Chambers, P.A., P. Lacoul, K.J., Murphy, S.M., (2010). World checklist of macrophyte species. Published on the internet; http://fada.biodiversity.be/group/show/60 accessed 10 August. 
[8]. Cook, C.D.K. (1996). Aquatic and wetland plants in India Oxford University press. London.

[9]. Gokhele. M.V., S.S.Shsikh and N.S. Chavan. (2011). Floaral survey of wet coastal and associated ecosystem to Maharastra. Indian journal of Geomarine Sciences. Vol. 40 (5) pp 725-730.

[10]. James e. Fulmer And T. Robinson (2006). Aquatic Plant Species Distributions and Associations in Arizona's Reservoirs. J. Aquat. Plant Manage. 46: 100-106.

[11]. Jeppesen, E., Søndergaard, M., Søndergaard, M., Christoffersen, K. (Eds.) (1998). The Structuring Role of Submerged Macrophytes in Lakes. Ecological Series, vol. 131. Springer-Verlag, 423 pp.

[12]. Kayode, J. and Ogunleye, O.T. (2008) Checklist and Status of Plant Species Used as Spices in Kaduna State of Nigeria. African Journal of General Agriculture 4, 13-18.

[13]. Kiran, B.R., Patel A.N., Kumar Vijaya and Puttaiah E.T. (2006). Aquatic macrophytes in fish culture ponds at Bhadra fish farm, Karnataka. J. Aqua.Biol. 21(2): 27-30.

[14]. Krull, J.N. (1970). Aquatic plant-invertebrate associations and waterfowl. Journal of Wildlife Management 34:707-718.

[15]. Kumar, M. and Singh, J. (1987) Environmental impacts of Aquatic Weeds and their classification. Proceedings of the workshop on management of Aquatic Weeds, Amritsar, Punjab, India.

[16]. Madsen, J.D., P.A. Chambers, W.F. James, E.W. Koch, and D.F. Westlake. (2001). The Interactions between water movement, sediment dynamics and submersed macrophytes. Hydrobiologia 444:71-84.

[17]. Majid,F.Z. (1986). Aquatic Weeds -Utility and Development, Agro Botanical Publishers, India.

[18]. Meshram, C. B. (2003). Macro invertebrate fauna of lake Wadali, Amaravati, Maharashtra., J. Aqua. Biol. Vol. 18(2): 47 - 50.

[19]. Naskar, K.R. (1990). Aquatic and Semi-aquatic Plants of the Lower Ganga Delta. Daya Publishing House, New Delhi.

[20]. Olsen, S. (1950). Aquatic plants and hydrospheric factor, I. Aquatic plants in Switzerland, Arizona. J. Sevensk. Botanisk Tidskriff, 44, 1-34.

[21]. Patil V.G., Khabade S.A. and Khade S.K. (2012). Study of hydrophytes and amphibious plants occurred in Panchganga River in vicinity of Ichalkaranji city district Kolhapur, Maharashta. Ecology and Fisheries 5(2): 63-66.

[22]. [22] Rama Rao. K. (2014 a). Diversity of Ornamental Fishes in Lower Manair Dam at Karimnagar Dt. Andhra Pradesh. IOSR Journal of Pharmacy and Biological Sciences (IOSR-JPBS) e-ISSN: 2278-3008, p-ISSN:2319-7676. Volume 9, Issue 1 Ver. I PP $20-24$.

[23]. Rama Rao K. (2014 b). A study on larvivorous fish species efficacy of lower Manair dam at Karimnagar, Andhra Pradesh, India. Pelagia Research Library. Advances in Applied Science Research, 5(2):133-143.

[24]. Raut, Nayana. S. and Pejaver Madhuri. (2005). Survey of diversity of plankton attached to macrophytes from weed infested lakes. J. Aqua. Biol. 20 (1): 1 - 7.

[25]. Shashikant R.Sitre., (2013). Assessment of Macrophyte Bio-Diversity of a Freshwater Reservoir of Bhadrawati Tehsil in Chandrapur District. Online International Interdisciplinary Research Journal, \{Bi-Monthly\}, ISSN2249-9598, Volume-III, Issue-III.

[26]. Spence, D.H.N. (1964). The macrophytes vegetation of lochs, swamps and associated fens. The vegetation of Scotland (Ed.: J.H. Burnett). Edinburgh. pp. 306-425.

[27]. Thomaz, S.M., L.M. Bini, M.C., Souza, K.K. Kita and Camargo. (1999). Aquatic Macrophytes of Itaipu Reservoir, Brazil: Survey of Species and Ecological Considerations. Braz Arch Biol.Techn 42: 15-22.

[28]. Wetzel, R.G. (1975). Limnology, W.B.Saunders Company, Philadelphia, pp.743.

Table: I. Checklist of free floating weeds of their scientific name, common name, class, order, family, habitat and IUCN red list at Lower manair Dam.

\begin{tabular}{|c|c|c|c|c|c|c|c|}
\hline S. No & $\begin{array}{l}\text { Name of } \\
\text { Species }\end{array}$ & $\begin{array}{l}\text { Common } \\
\text { name/s }\end{array}$ & Class & Order & Family & Habitat & $\begin{array}{c}\text { IUCN } \\
(2013.2 \\
)\end{array}$ \\
\hline 1 & $\begin{array}{l}1 . \quad \text { A } \\
\text { zolla } \\
\text { filiculoides }\end{array}$ & Water Fern & $\begin{array}{l}\text { Polypodio } \\
\text { psida }\end{array}$ & $\begin{array}{l}\text { Salvinial } \\
\text { es }\end{array}$ & $\begin{array}{l}\text { Salvinia } \\
\text { ceae }\end{array}$ & $\begin{array}{l}\text { It is most frequent in ponds, lakes, } \\
\text { canals, ditches and slow flowing } \\
\text { rivers. }\end{array}$ & $\mathrm{NE}$ \\
\hline 2 & $\begin{array}{l}\text { Eichornia } \\
\text { crassipes } \\
\text { (Mart.) } \\
\text { Solms. }\end{array}$ & $\begin{array}{l}\text { Water } \\
\text { hyacinth }\end{array}$ & Liliopsida & $\begin{array}{l}\text { Commel } \\
\text { inales }\end{array}$ & $\begin{array}{l}\text { Ponteder } \\
\text { iaceae }\end{array}$ & $\begin{array}{l}\text { Shallow temporary ponds, } \\
\text { wetlands and marshes, sluggish } \\
\text { flowing waters, lakes, reservoirs } \\
\text { and rivers }\end{array}$ & $\mathrm{NE}$ \\
\hline 3 & $\begin{array}{l}\text { Lemna } \\
\text { perpusilla } \\
\text { Torrey } \\
\end{array}$ & Duckweed & Liliopsida & Arales & $\begin{array}{l}\text { Lemnac } \\
\text { eae }\end{array}$ & $\begin{array}{l}\text { It grows in paddy fields, ponds and } \\
\text { other still water bodies. }\end{array}$ & $\mathrm{LC}$ \\
\hline 4 & $\begin{array}{l}\text { Ottelia } \\
\text { alismoides } \\
\text { (L.) Pers. }\end{array}$ & $\begin{array}{l}\text { Duck- } \\
\text { lettuce, } \\
\text { waterplantai } \\
\text { n ottelia } \\
\end{array}$ & Liliopsida & $\begin{array}{l}\text { Hydroch } \\
\text { aritales }\end{array}$ & $\begin{array}{l}\text { Hydroch } \\
\text { aritaceae }\end{array}$ & $\begin{array}{l}\text { This species grows in shallow } \\
\text { water edges and ponds and in rice } \\
\text { field }\end{array}$ & $\mathrm{LC}$ \\
\hline 5 & $\begin{array}{l}\text { Pistia } \\
\text { stratiotes L. }\end{array}$ & $\begin{array}{l}\text { Tropical } \\
\text { duck-weed, } \\
\text { water } \\
\text { lettuce }\end{array}$ & Liliopsida & Arales & Araceae & $\begin{array}{l}\text { It is a free-floating weed and } \\
\text { capable of forming dense mats on } \\
\text { the surfaces of lakes, ponds, rivers } \\
\text { and other water bodies. }\end{array}$ & $\mathrm{LC}$ \\
\hline
\end{tabular}


Table: II. Checklist of Submerged weeds of their scientific name, common name, class, order, family, habitat and IUCN red list at Lower manair Dam.

\begin{tabular}{|c|c|c|c|c|c|c|c|}
\hline S.No & Name of Species & $\begin{array}{c}\text { Common } \\
\text { Name/s } \\
\end{array}$ & Class & Order & $\begin{array}{c}\text { Famil } \\
\mathbf{y}\end{array}$ & Habitat & $\begin{array}{l}\text { IUCN } \\
2013.2 \\
\end{array}$ \\
\hline 1 & $\begin{array}{l}\text { Aponogeton natans } \\
\text { L.f }\end{array}$ & $\begin{array}{l}\text { Floating lace } \\
\text { Plant, Drifting } \\
\text { Sword Plant }\end{array}$ & Liliopsida & $\begin{array}{l}\text { Najadal } \\
\text { es }\end{array}$ & $\begin{array}{l}\text { Apono } \\
\text { getona } \\
\text { ceae }\end{array}$ & $\begin{array}{l}\text { It grows in seasonal } \\
\text { and permanent still or } \\
\text { flowing waters, rice } \\
\text { fields and marshy } \\
\text { places. }\end{array}$ & $\mathrm{LC}$ \\
\hline 2 & $\begin{array}{l}\text { Chara globularis J. } \\
\text { L.T }\end{array}$ & $\begin{array}{l}\text { Muskgrass, } \\
\text { stonewort, } \\
\text { muskwort }\end{array}$ & $\begin{array}{l}\text { Charophy } \\
\text { ceae }\end{array}$ & $\begin{array}{l}\text { Charale } \\
\text { s }\end{array}$ & $\begin{array}{l}\text { Charac } \\
\text { eae }\end{array}$ & $\begin{array}{l}\text { It is found both in } \\
\text { freshwater and } \\
\text { brackish water. In } \\
\text { freshwater it is found } \\
\text { in lakes and rivers, } \\
\text { oligotrophic as well as } \\
\text { eutrophic water. It } \\
\text { grows on sand, clay, } \\
\text { mud or marl bottom. }\end{array}$ & $\mathrm{NE}$ \\
\hline 3 & $\begin{array}{l}\text { Ceratophyllum } \\
\text { demersum L. }\end{array}$ & $\begin{array}{l}\text { Rigid Hornwort } \\
\text {, Coontail, } \\
\text { Hornwort }\end{array}$ & $\begin{array}{l}\text { Magnolio } \\
\text { psida }\end{array}$ & $\begin{array}{l}\text { Nymph } \\
\text { aeales }\end{array}$ & $\begin{array}{l}\text { Cerato } \\
\text { phyllac } \\
\text { eae }\end{array}$ & $\begin{array}{l}\text { Less saline parts of sea } \\
\text { inlets, ponds, slow- } \\
\text { flowing streams }\end{array}$ & $\mathrm{LC}$ \\
\hline 4 & $\begin{array}{l}\text { Hydrilla verticillata } \\
\text { (L. f.) Royle }\end{array}$ & $\begin{array}{l}\text { Florida-elodea, } \\
\text { Indian } \\
\text { Stargrass, } \\
\text { Water-thyme }\end{array}$ & Liliopsida & $\begin{array}{l}\text { Hydroc } \\
\text { haritale } \\
\mathrm{s}\end{array}$ & $\begin{array}{l}\text { Hydro } \\
\text { charita } \\
\text { ceae }\end{array}$ & $\begin{array}{l}\text { Rrivers and ponds. } \\
\text { This species is hardy } \\
\text { and tolerant to heavy } \\
\text { metals. }\end{array}$ & $\mathrm{LC}$ \\
\hline 5 & $\begin{array}{l}\text { Ipomoea aquatica } \\
\text { Forsk (Rooted) }\end{array}$ & Water spinach & $\begin{array}{l}\text { Magnolio } \\
\text { psida }\end{array}$ & $\begin{array}{l}\text { Solanal } \\
\text { es }\end{array}$ & $\begin{array}{l}\text { Convol } \\
\text { vulace } \\
\text { ae }\end{array}$ & $\begin{array}{l}\text { Found in marshy } \\
\text { habitats and ditches, } \\
\text { muddy stream banks, } \\
\text { ponds, lakes, rice } \\
\text { paddies and waste } \\
\text { areas. }\end{array}$ & $\mathrm{LC}$ \\
\hline 6 & $\begin{array}{l}\text { Ipomoea carnea } \\
\text { jacq }\end{array}$ & $\begin{array}{l}\text { Pink morning } \\
\text { glory }\end{array}$ & $\begin{array}{l}\text { Magnolio } \\
\text { psida }\end{array}$ & $\begin{array}{l}\text { Solanal } \\
\text { es }\end{array}$ & $\begin{array}{l}\text { Convol } \\
\text { vulace } \\
\text { ae }\end{array}$ & $\begin{array}{l}\text { Grows in } r \text { dense } \\
\text { populations along river } \\
\text { beds, river banks, } \\
\text { canals and other } \\
\text { waterlogged (wetland) } \\
\text { areas. It has become } \\
\text { naturalized along } \\
\text { canals, drains and } \\
\text { road sides }\end{array}$ & $\mathrm{NE}$ \\
\hline 7 & Marsilea quadrifolia & $\begin{array}{l}\text { Water } \\
\text { Shamrock }\end{array}$ & $\begin{array}{l}\text { Polypodio } \\
\text { psida }\end{array}$ & $\begin{array}{l}\text { Salvini } \\
\text { ales }\end{array}$ & $\begin{array}{l}\text { Marsil } \\
\text { eaceae }\end{array}$ & $\begin{array}{l}\text { It grows in still waters } \\
\text { such as ponds, rice } \\
\text { fields and ditches. }\end{array}$ & $\mathrm{LC}$ \\
\hline 8 & Najas minor L. & Slender Naiad & Liliopsida & $\begin{array}{l}\text { Hydroc } \\
\text { haritale } \\
\mathrm{s}\end{array}$ & $\begin{array}{l}\text { Hydro } \\
\text { charita } \\
\text { ceae }\end{array}$ & $\begin{array}{l}\text { Ponds, lakes, rivers, } \\
\text { streams, ditches and } \\
\text { paddy fields. }\end{array}$ & $\mathrm{LC}$ \\
\hline 9 & $\begin{array}{l}\text { Potamogeton } \\
\text { pectinata L. }\end{array}$ & $\begin{array}{l}\text { Fennel } \\
\text { Pondweed }\end{array}$ & Liliopsida & $\begin{array}{l}\text { Najadal } \\
\text { es }\end{array}$ & $\begin{array}{l}\text { Potam } \\
\text { ogeton } \\
\text { aceae }\end{array}$ & $\begin{array}{l}\text { Typical of eutrophic or } \\
\text { brackish waters } \\
\text { lakes, reservoirs, } \\
\text { rivers, streams, canals, } \\
\text { ditches, pond }\end{array}$ & $\mathrm{LC}$ \\
\hline 10 & $\begin{array}{l}\text { Vallisneria spiralis } \\
\text { L. }\end{array}$ & Tapegrass & Liliopsida & $\begin{array}{l}\text { Hydroc } \\
\text { haritale } \\
\text { s }\end{array}$ & $\begin{array}{l}\text { Hydro } \\
\text { charita } \\
\text { ceae }\end{array}$ & $\begin{array}{l}\text { Estuarine habitats, } \\
\text { lakes, rivers, } \\
\text { waterlogged swamps } \\
\text { with open water, man- } \\
\text { made reservoirs, }\end{array}$ & $\mathrm{LC}$ \\
\hline
\end{tabular}

Table: III. Checklist of emergent weeds of their Scientific name, common name, class, order, family, habitat and IUCN red list at Lower Manair Dam.

\begin{tabular}{|c|c|c|c|c|c|c|c|}
\hline S.No & Name of Species & Common Name/s & Class & Order & Family & Habitat & $\begin{array}{l}\text { IUCN } \\
2013.2\end{array}$ \\
\hline 1 & $\begin{array}{l}\text { Alternanthera } \\
\text { sessilis (L.)R. Br. ex }\end{array}$ & $\begin{array}{l}\text { Sessile joyweed, } \\
\text { dwarf copperleaf }\end{array}$ & $\begin{array}{l}\text { Magnoli } \\
\text { opsida }\end{array}$ & $\begin{array}{l}\text { Caryo } \\
\text { phyllal } \\
\text { es }\end{array}$ & $\begin{array}{l}\text { Amaran } \\
\text { thaceae }\end{array}$ & $\begin{array}{l}\text { Aquatic and wetland } \\
\text { habitats } \\
\text { It may extend into } \\
\text { brackish water and } \\
\text { estuarine habitats near } \\
\text { the coast. }\end{array}$ & $\mathrm{LC}$ \\
\hline 2 & $\begin{array}{l}\text { Alternanthera } \\
\text { philoxeroides }\end{array}$ & $\begin{array}{l}\text { Sessile joyweed, } \\
\text { dwarf copperleaf }\end{array}$ & $\begin{array}{l}\text { Magnoli } \\
\text { opsida }\end{array}$ & $\begin{array}{l}\text { Caryo } \\
\text { phyllal } \\
\text { es }\end{array}$ & $\begin{array}{l}\text { Amaran } \\
\text { thaceae }\end{array}$ & $\begin{array}{l}\text { Aquatic and wetland } \\
\text { plants. }\end{array}$ & $\mathrm{LC}$ \\
\hline 3 & $\begin{array}{l}\text { Ammannia } \\
\text { baccifera L. }\end{array}$ & Blistering ammania & $\begin{array}{l}\text { Magnoli } \\
\text { opsida }\end{array}$ & $\begin{array}{l}\text { Myrtal } \\
\text { es }\end{array}$ & $\begin{array}{l}\text { Lythrac } \\
\text { eae }\end{array}$ & $\begin{array}{l}\text { It is an annual } \\
\text { herbaceous }\end{array}$ & $\mathrm{LC}$ \\
\hline
\end{tabular}


Checklist Of The Aquatic Macrophyte Flora Abundence In Lower Manair Dam At Karimnagar ....

\begin{tabular}{|c|c|c|c|c|c|c|c|}
\hline & & & & & & $\begin{array}{l}\text { (therophyte) that is found } \\
\text { in swampy areas, rice } \\
\text { paddies and freshwater }\end{array}$ & \\
\hline 4 & $\begin{array}{l}\text { Aeschynomene } \\
\text { indica L. }\end{array}$ & India joint-vetch & $\begin{array}{l}\text { Magnoli } \\
\text { opsida }\end{array}$ & $\begin{array}{l}\text { Fabale } \\
\mathrm{s}\end{array}$ & $\begin{array}{l}\text { Fabacea } \\
\text { e }\end{array}$ & $\begin{array}{l}\text { Grows in field margins, } \\
\text { ditches, grasslands, trail } \\
\text { sides and river margins }\end{array}$ & $\mathrm{LC}$ \\
\hline 5 & $\begin{array}{l}\text { Bacopa monnieri } \\
\text { (L.) Wettestin }\end{array}$ & Water hyssop & $\begin{array}{l}\text { Magnoli } \\
\text { opsida }\end{array}$ & $\begin{array}{l}\text { Scroph } \\
\text { ularial } \\
\text { es }\end{array}$ & $\begin{array}{l}\text { Scrophu } \\
\text { lariacea } \\
\text { e }\end{array}$ & $\begin{array}{l}\text { Forms dense mats in } \\
\text { marshy places, the banks } \\
\text { of pools and along } \\
\text { streams and ditches. }\end{array}$ & LC \\
\hline 6 & Coix aquatica Roxb. & Aquatic Job's tears & $\begin{array}{l}\text { Liliopsi } \\
\text { da }\end{array}$ & Poales & Poaceae & $\begin{array}{l}\text { It is a perennial, aquatic } \\
\text { herb. Stems (culms) are } \\
\text { creeping and rooting } \\
\text { from nodes at base, } \\
\text { sometimes floating }\end{array}$ & $\mathrm{NE}$ \\
\hline 7 & $\begin{array}{l}\text { Commelina } \\
\text { benghalensis } \mathrm{L} \text {. }\end{array}$ & Bengal day flower & $\begin{array}{l}\text { Liliopsi } \\
\text { da }\end{array}$ & $\begin{array}{l}\text { Comm } \\
\text { elinale } \\
\text { s }\end{array}$ & $\begin{array}{l}\text { Comme } \\
\text { linaceae }\end{array}$ & $\begin{array}{l}\text { A widespread weed often } \\
\text { found in ditches, wet } \\
\text { fields and places }\end{array}$ & $\mathrm{LC}$ \\
\hline 8 & $\begin{array}{l}\text { Commelina } \\
\text { hasskarlii C. Comm. } \\
\text { Cyrt. }\end{array}$ & Carolina dayflower & $\begin{array}{l}\text { Liliopsi } \\
\text { da }\end{array}$ & $\begin{array}{l}\text { Comm } \\
\text { elinale } \\
\mathrm{s}\end{array}$ & $\begin{array}{l}\text { Comme } \\
\text { linaceae }\end{array}$ & $\begin{array}{l}\text { Commonly found in } \\
\text { fields, swamps, yards, } \\
\text { waste places, along } \\
\text { roadsides, rarely in the } \\
\text { forests. }\end{array}$ & $\mathrm{LC}$ \\
\hline 9 & Cyperus rotundus L. & Nut-grass & $\begin{array}{l}\text { Liliopsi } \\
\text { da }\end{array}$ & $\begin{array}{l}\text { Cypera } \\
\text { les }\end{array}$ & $\begin{array}{l}\text { Cyperac } \\
\text { eae }\end{array}$ & $\begin{array}{l}\text { Occurs in a wide variety } \\
\text { of wetland habitats } \\
\text { including seasonally wet } \\
\text { grasslands, swamps, } \\
\text { ditches, pond and lake } \\
\text { margins, springs, stream } \\
\text { and river banks. }\end{array}$ & $\mathrm{LC}$ \\
\hline 10 & Cyperus difformis L & $\begin{array}{l}\text { Smallflower } \\
\text { Umbrella Sedge }\end{array}$ & $\begin{array}{l}\text { Liliopsi } \\
\text { da }\end{array}$ & $\begin{array}{l}\text { Cypera } \\
\text { les }\end{array}$ & $\begin{array}{l}\text { Cyperac } \\
\text { eae }\end{array}$ & $\begin{array}{l}\text { The plant normally } \\
\text { grows in flooded or in } \\
\text { very moist soils. It is } \\
\text { frequently found in small } \\
\text { pools, along rivers, } \\
\text { canals, and streams, in } \\
\text { open wet places, and in } \\
\text { grassy swamps. }\end{array}$ & $\mathrm{LC}$ \\
\hline 11 & $\begin{array}{l}\text { Cynodon dactylon } \\
\text { (L.) Pers. }\end{array}$ & $\begin{array}{l}\text { Bermuda } \\
\text { grass, Indian doab, } \\
\text { devil's grass. }\end{array}$ & $\begin{array}{l}\text { Liliopsi } \\
\text { da }\end{array}$ & Poales & Poaceae & Water courses, wetlands & LC \\
\hline 12 & $\begin{array}{l}\text { Cyathocline } \\
\text { purpurea (Buch- } \\
\text { Ham. ex D. Don) } \\
\text { Oktze }\end{array}$ & Purple bane & $\begin{array}{l}\text { Magnoli } \\
\text { opsida }\end{array}$ & $\begin{array}{l}\text { Asteral } \\
\text { es }\end{array}$ & $\begin{array}{l}\text { Asterac } \\
\text { eae }\end{array}$ & $\begin{array}{l}\text { It is found growing in } \\
\text { water logged soils } \\
\text { subjected to seasonal } \\
\text { inundation and along } \\
\text { waterways }\end{array}$ & $\mathrm{LC}$ \\
\hline 13 & $\begin{array}{l}\text { Chrozophora rottleri } \\
\text { (Geisel.) A. Juss. ex. } \\
\text { Spr }\end{array}$ & Suryavarti & $\begin{array}{l}\text { Magnoli } \\
\text { opsida }\end{array}$ & $\begin{array}{l}\text { Malpig } \\
\text { hiales }\end{array}$ & $\begin{array}{l}\text { Euphor } \\
\text { biaceae }\end{array}$ & $\begin{array}{l}\text { Paddy fields and } \\
\text { reservoir banks }\end{array}$ & $\mathrm{NE}$ \\
\hline 14 & $\begin{array}{l}\text { Dopatrium junceum } \\
\text { (Roxb.) Buch- Hum } \\
\text { ex Benth. }\end{array}$ & Rushlike dopatrium & $\begin{array}{l}\text { Magnoli } \\
\text { opsida }\end{array}$ & $\begin{array}{l}\text { Scroph } \\
\text { ularial } \\
\text { es }\end{array}$ & $\begin{array}{l}\text { Scrophu } \\
\text { lariacea } \\
\text { e }\end{array}$ & $\begin{array}{l}\text { It grows in post-monsoon } \\
\text { pools, rice fields and } \\
\text { perennially wet places } \\
\text { below } 1,800 \mathrm{~m}\end{array}$ & $\mathrm{LC}$ \\
\hline 15 & Eclipta alba & $\begin{array}{l}\text { False Daisy, } \\
\text { Bhringraj. }\end{array}$ & $\begin{array}{l}\text { Magnoli } \\
\text { opsida }\end{array}$ & $\begin{array}{l}\text { Asteral } \\
\text { es }\end{array}$ & $\begin{array}{l}\text { Asterac } \\
\text { eae }\end{array}$ & $\begin{array}{l}\text { Grows commonly in } \\
\text { moist places as a weed in } \\
\text { warm temperate to } \\
\text { tropical areas worldwide. }\end{array}$ & LC \\
\hline 16 & $\begin{array}{l}\text { Eleocharis } \\
\text { geniculata (L.) } \\
\text { R\&S. }\end{array}$ & $\begin{array}{l}\text { Canada spikesedge, } \\
\text { spike rush }\end{array}$ & $\begin{array}{l}\text { Liliopsi } \\
\mathrm{da}\end{array}$ & $\begin{array}{l}\text { Cypera } \\
\text { les }\end{array}$ & $\begin{array}{l}\text { Cyperac } \\
\text { eae }\end{array}$ & $\begin{array}{l}\text { It is an annual herb, } \\
\text { potently grows in } \\
\text { clumps, gregarious in } \\
\text { shallow water, } \\
\text { particularly common in } \\
\text { fallow rice fields, } \\
\text { sometimes in brackish } \\
\text { water by the sea and also } \\
\text { grows in wet sandy } \\
\text { localities }\end{array}$ & LC \\
\hline 17 & $\begin{array}{l}\text { Eleocharis capitata } \\
\text { R. Br. }\end{array}$ & Spike-rush & $\begin{array}{l}\text { Liliopsi } \\
\text { da }\end{array}$ & $\begin{array}{l}\text { Cypera } \\
\text { les }\end{array}$ & $\begin{array}{l}\text { Cyperac } \\
\text { eae }\end{array}$ & $\begin{array}{l}\text { Wetlands, banks of pools } \\
\text { and streams, terrestrial; } \\
\text { freshwater }\end{array}$ & LC \\
\hline 18 & Eriocaulon & Pipewort, & Liliopsi & Poales & Eriocau & Damp shady place. Rice & $\mathrm{NE}$ \\
\hline
\end{tabular}


Checklist Of The Aquatic Macrophyte Flora Abundence In Lower Manair Dam At Karimnagar ....

\begin{tabular}{|c|c|c|c|c|c|c|c|}
\hline & cinereum R.Br. & & da & & laceae & $\begin{array}{l}\text { fields, valleys, and damp } \\
\text { soils from near sea level }\end{array}$ & \\
\hline 19 & $\begin{array}{l}\text { Echinochloa colona } \\
\text { (L.) Link }\end{array}$ & Wild grass & $\begin{array}{l}\text { Liliopsi } \\
\text { da }\end{array}$ & Poales & Poaceae & $\begin{array}{l}\text { Wetland habitats such as } \\
\text { rice fields, roadsides, } \\
\text { river banks, shores of } \\
\text { ponds, the inner edges of } \\
\text { mangrove swamps and } \\
\text { old clearings. }\end{array}$ & $\mathrm{LC}$ \\
\hline 20 & $\begin{array}{l}\text { Fimbristylis } \\
\text { miliacea }\end{array}$ & $\begin{array}{l}\text { Lesser Fimbristylis, } \\
\text { Grass-Like } \\
\text { Fimbristylis }\end{array}$ & $\begin{array}{l}\text { Liliopsi } \\
\mathrm{da}\end{array}$ & $\begin{array}{l}\text { Cypera } \\
\text { les }\end{array}$ & $\begin{array}{l}\text { Cyperac } \\
\text { eae }\end{array}$ & $\begin{array}{l}\text { It is an annual or } \\
\text { perennial herb growing } \\
\text { in wet places, ponds, } \\
\text { streams and at the edges } \\
\text { of drying pools. }\end{array}$ & LC \\
\hline 21 & $\begin{array}{l}\text { Gomphrena } \\
\text { celosioides Mart. }\end{array}$ & Gomphrena weed & $\begin{array}{l}\text { magnoli } \\
\text { opsida }\end{array}$ & $\begin{array}{l}\text { Caryo } \\
\text { phyllal } \\
\text { es }\end{array}$ & $\begin{array}{l}\text { Amaran } \\
\text { thaceae }\end{array}$ & $\begin{array}{l}\text { A common plant of } \\
\text { roadsides, and sometimes } \\
\text { invading pastures as a } \\
\text { weed }\end{array}$ & $\mathrm{NE}$ \\
\hline 22 & Glinus lotoides L. & Lotus sweetjuice & $\begin{array}{l}\text { magnoli } \\
\text { opsida }\end{array}$ & $\begin{array}{l}\text { Caryo } \\
\text { phyllal } \\
\text { es }\end{array}$ & $\begin{array}{l}\text { Mollugi } \\
\text { naceae }\end{array}$ & Lake, marsh & $\mathrm{NE}$ \\
\hline 23 & $\begin{array}{l}\text { Gnaphalium } \\
\text { pulvinatum Del. }\end{array}$ & pinyin & $\begin{array}{l}\text { Magnoli } \\
\text { opsida }\end{array}$ & $\begin{array}{l}\text { Asteral } \\
\text { es }\end{array}$ & $\begin{array}{l}\text { Asterac } \\
\text { eae }\end{array}$ & $\begin{array}{l}\text { On low land which has } \\
\text { been flooded. }\end{array}$ & $\mathrm{NE}$ \\
\hline 24 & $\begin{array}{l}\text { Grangea } \\
\text { maderaspatana }\end{array}$ & Madras Carpet & $\begin{array}{l}\text { Magnoli } \\
\text { opsida }\end{array}$ & $\begin{array}{l}\text { Asteral } \\
\text { es }\end{array}$ & $\begin{array}{l}\text { Asterac } \\
\text { eae }\end{array}$ & $\begin{array}{l}\text { The species occurs on } \\
\text { the edges of ponds, } \\
\text { canals and ditches, as } \\
\text { well as rice fields. }\end{array}$ & LC \\
\hline 25 & $\begin{array}{l}\text { Heliotropium } \\
\text { supinum L. }\end{array}$ & Dwarf heliotrope & $\begin{array}{l}\text { Magnoli } \\
\text { opsida }\end{array}$ & $\begin{array}{l}\text { Boragi } \\
\text { nales }\end{array}$ & $\begin{array}{l}\text { Boragin } \\
\text { aceae }\end{array}$ & $\begin{array}{l}\text { Sandy and alluvial } \\
\text { plains, waste ground, } \\
\text { edges of cultivation. }\end{array}$ & $\mathrm{LC}$ \\
\hline 26 & $\begin{array}{l}\text { Limnophila } \\
\text { sessiliflora L. }\end{array}$ & Asian marshweed & $\begin{array}{l}\text { Magnoli } \\
\text { opsida }\end{array}$ & $\begin{array}{l}\text { Scroph } \\
\text { ularial } \\
\text { es }\end{array}$ & $\begin{array}{l}\text { Scrophu } \\
\text { lariacea } \\
\text { e }\end{array}$ & $\begin{array}{l}\text { Wetland habitats, } \\
\text { including the margins of } \\
\text { ponds, swamps, rice } \\
\text { fields, seasonally } \\
\text { inundated habitats along } \\
\text { streams and low-lying } \\
\text { wet areas. }\end{array}$ & LC \\
\hline 27 & $\begin{array}{l}\text { Polygonum glabrum } \\
\text { Willd. }\end{array}$ & Marsh buckwheat & $\begin{array}{l}\text { magnoli } \\
\text { opsida }\end{array}$ & $\begin{array}{l}\text { Polygo } \\
\text { nales }\end{array}$ & $\begin{array}{l}\text { Polygon } \\
\text { aceae }\end{array}$ & $\begin{array}{l}\text { Common, } \\
\text { gregariously in marshy } \\
\text { areas. Plains }\end{array}$ & $\mathrm{NE}$ \\
\hline 28 & $\begin{array}{l}\text { Phyla nodiflora (L.) } \\
\text { Greene }\end{array}$ & $\begin{array}{l}\text { Turkey tangle } \\
\text { frogfruit }\end{array}$ & $\begin{array}{l}\text { Magnoli } \\
\text { opsida }\end{array}$ & $\begin{array}{l}\text { Lamial } \\
\text { es }\end{array}$ & $\begin{array}{l}\text { Verben } \\
\text { aceae }\end{array}$ & $\begin{array}{l}\text { A marshy herb which } \\
\text { grows in open and wet } \\
\text { places near streams, } \\
\text { ponds, paddy fields, } \\
\text { ditches, backwaters, } \\
\text { brackish water }\end{array}$ & LC \\
\hline 29 & $\begin{array}{l}\text { Rotala serpyllifolia } \\
\text { (Roth.) Bremeck }\end{array}$ & Rotala & $\begin{array}{l}\text { Magnoli } \\
\text { opsida }\end{array}$ & $\begin{array}{l}\text { Myrtal } \\
\text { es }\end{array}$ & $\begin{array}{l}\text { Lythrac } \\
\text { eae }\end{array}$ & $\begin{array}{l}\text { It grows submerged in } \\
\text { rivers, streams and lakes } \\
\text { but is also found in } \\
\text { standing water recedes. }\end{array}$ & LC \\
\hline 30 & $\begin{array}{l}\text { Sesbania bispinosa } \\
\text { (Jacq.) w. t. wight }\end{array}$ & Sesbania pea & $\begin{array}{l}\text { Magnoli } \\
\text { opsida }\end{array}$ & $\begin{array}{l}\text { Fabale } \\
\mathrm{s}\end{array}$ & $\begin{array}{l}\text { Fabacea } \\
\text { e }\end{array}$ & $\begin{array}{l}\text { It is not confined to } \\
\text { wetlands but often found } \\
\text { in swamps, marshy } \\
\text { wastelands, water-logged } \\
\text { places, banks of ponds } \\
\text { and rivers, rice fields and } \\
\text { seasonally inundated } \\
\text { areas. }\end{array}$ & LC \\
\hline 31 & $\begin{array}{l}\text { Sopubia } \\
\text { delphinifolia (L.) G. } \\
\text { Don }\end{array}$ & $\begin{array}{l}\text { Sopubia } \\
\text { delphinifolia }\end{array}$ & $\begin{array}{l}\text { Magnoli } \\
\text { opsida }\end{array}$ & $\begin{array}{l}\text { Scroph } \\
\text { ularial } \\
\text { es }\end{array}$ & $\begin{array}{l}\text { Scrophu } \\
\text { lariacea } \\
\text { e }\end{array}$ & $\begin{array}{l}\text { Western India, as a root } \\
\text { parasite. }\end{array}$ & $\overline{\mathrm{VU}}$ \\
\hline 32 & $\begin{array}{l}\text { Sphaeranthus } \\
\text { indicus L. }\end{array}$ & $\begin{array}{l}\text { East indian globe } \\
\text { thistle }\end{array}$ & $\begin{array}{l}\text { Magnoli } \\
\text { opsida }\end{array}$ & $\begin{array}{l}\text { Asteral } \\
\text { es }\end{array}$ & $\begin{array}{l}\text { Asterac } \\
\text { eae }\end{array}$ & $\begin{array}{l}\text { It is an annual found } \\
\text { growing in wet areas, } \\
\text { occasionally temporarily } \\
\text { submerged. Common in } \\
\text { and around irrigation } \\
\text { ditches }\end{array}$ & LC \\
\hline 33 & $\begin{array}{l}\text { Typha angustata } \\
\text { Bory and Chaub. }\end{array}$ & $\begin{array}{l}\text { Reed Mace, } \\
\text { Elephant Grass, }\end{array}$ & $\begin{array}{l}\text { Liliopsi } \\
\mathrm{da}\end{array}$ & $\begin{array}{l}\text { Typhal } \\
\text { es }\end{array}$ & $\begin{array}{l}\text { Typhac } \\
\text { eae }\end{array}$ & $\begin{array}{l}\text { It occurs in shallow } \\
\text { water of lakes, ponds, } \\
\text { rivers, swamps and } \\
\text { channels. }\end{array}$ & $\mathrm{NE}$ \\
\hline
\end{tabular}


Checklist Of The Aquatic Macrophyte Flora Abundence In Lower Manair Dam At Karimnagar ....

Table: 4. Number of classes, orders, families and species of Macrophytes at lower Manair Dam.

\begin{tabular}{|l|c|c|c|c|}
\hline Classification & Total species & Free floating weeds & Submerged weeds & Emergent weeds \\
\hline Class & 04 & 02 & 04 & 02 \\
\hline Order & 20 & 04 & 06 & 13 \\
\hline Family & 26 & 05 & 06 & 15 \\
\hline Species & 48 & 05 & 10 & 33 \\
\hline
\end{tabular}

Table: 5. Number and Percentage contribution of Macrophytes in Four classes.

\begin{tabular}{|l|c|c|c|c|c|c|}
\hline \multirow{2}{*}{ Class (4) } & \multicolumn{2}{|c|}{ Free floating } & \multicolumn{2}{c|}{ Submerged weeds } & \multicolumn{2}{c|}{ Emergent weeds } \\
\cline { 2 - 7 } & Total No.s & $\%$ contribution & Total No.s & $\%$ contribution & Total No.s & $\%$ contribution \\
\hline Liliopsida & 4 & 80.00 & 5 & 50.00 & 12 & 36.37 \\
\hline Polypodiopsida & 1 & 20.00 & 1 & 10.00 & 0 & 0 \\
\hline Charophyceae & 0 & 0 & 1 & 10.00 & 0 & 0 \\
\hline Magnoliopsida & 0 & 0 & 3 & 30.00 & 21 & 63.64 \\
\hline
\end{tabular}

Table: 6. Number and Percentage contribution of Macrophytes in Twenty orders.

\begin{tabular}{|c|c|c|c|c|c|c|c|c|}
\hline \multirow[b]{2}{*}{ Order (20) } & \multicolumn{2}{|c|}{ Free floating } & \multicolumn{2}{|c|}{ Submerged weeds } & \multicolumn{2}{|c|}{\begin{tabular}{l|l} 
Emergent weeds & \\
\end{tabular}} & \multicolumn{2}{|c|}{ Total } \\
\hline & $\begin{array}{l}\text { Total } \\
\text { No.s }\end{array}$ & $\begin{array}{l}\% \\
\text { contributi } \\
\text { on }\end{array}$ & Total No.s & $\begin{array}{l}\% \\
\text { contributio } \\
\mathrm{n}\end{array}$ & $\begin{array}{l}\text { Total } \\
\text { No.s }\end{array}$ & $\begin{array}{l}\% \\
\text { contribution }\end{array}$ & $\begin{array}{l}\text { Total } \\
\text { No.s }\end{array}$ & $\begin{array}{l}\% \\
\text { contributio } \\
\mathrm{n}\end{array}$ \\
\hline Arales & 02 & 40.00 & - & - & - & - & 02 & 4.17 \\
\hline Commelinales & 01 & 20.00 & - & - & 02 & 6.06 & 03 & 6.25 \\
\hline Hydrocharitales & 01 & 20.00 & 03 & 30.00 & - & - & 04 & 8.33 \\
\hline Salviniales & 01 & 20.00 & 01 & 10.00 & - & - & 02 & 4.17 \\
\hline Charales & - & - & 01 & 10.00 & - & - & 01 & 2.08 \\
\hline Najadales & - & - & 02 & 20.00 & - & - & 02 & 4.17 \\
\hline Nymphaeales & - & - & 01 & 10.00 & - & - & 01 & 2.08 \\
\hline Solanales & - & - & 02 & 20.00 & - & - & 02 & 4.17 \\
\hline Asterales & - & - & - & - & 05 & 15.15 & 05 & 10.42 \\
\hline Boraginales & - & - & - & - & 01 & 3.03 & 01 & 2.08 \\
\hline Caryophyllales & - & - & - & - & 04 & 12.12 & 04 & 8.33 \\
\hline Cyperales & - & - & - & - & 05 & 15.15 & 05 & 10.42 \\
\hline Fabales & - & - & - & - & 02 & 6.06 & 02 & 4.17 \\
\hline Lamiales & - & - & - & - & 01 & 3.03 & 01 & 2.08 \\
\hline Malpighiales & - & - & - & - & 01 & 3.03 & 01 & 2.08 \\
\hline Myrtales & - & - & - & - & 02 & 6.06 & 02 & 4.17 \\
\hline Poales & - & - & - & - & 04 & 12.12 & 04 & 8.33 \\
\hline Polygonales & - & - & - & - & 01 & 3.03 & 01 & 2.08 \\
\hline Scrophulariales & - & - & - & - & 04 & 12.12 & 04 & 8.33 \\
\hline Typhales & - & - & - & - & 01 & 3.03 & 01 & 2.08 \\
\hline Total & 05 & & 10 & & 33 & & 48 & \\
\hline
\end{tabular}

Table: 7. Number and Percentage contribution of Macrophyte in Twenty families.

\begin{tabular}{|c|c|c|c|c|c|c|c|c|}
\hline \multirow[t]{2}{*}{ Families (26) } & \multicolumn{2}{|c|}{ Free floating } & \multicolumn{2}{|c|}{ Submerged weeds } & \multicolumn{2}{|c|}{ Emergent weeds } & \multicolumn{2}{|c|}{ Total } \\
\hline & $\begin{array}{l}\text { Total } \\
\text { No.s }\end{array}$ & $\begin{array}{l}\% \\
\text { contribution }\end{array}$ & $\begin{array}{l}\text { Total } \\
\text { No.s }\end{array}$ & $\begin{array}{l}\% \\
\text { contribution }\end{array}$ & $\begin{array}{l}\text { Total } \\
\text { No.s }\end{array}$ & $\begin{array}{l}\% \\
\text { contribution }\end{array}$ & $\begin{array}{l}\text { Total } \\
\text { No.s }\end{array}$ & $\begin{array}{l}\% \\
\text { contribution }\end{array}$ \\
\hline Araceae & 1 & 20.00 & - & - & - & - & 1 & 2.08 \\
\hline Hydrocharitaceae & 1 & 20.00 & 3 & 30.00 & - & - & 4 & 8.33 \\
\hline Lemnaceae & 1 & 20.00 & - & - & - & - & 1 & 2.08 \\
\hline Pontederiaceae & 1 & 20.00 & - & - & - & - & 1 & 2.08 \\
\hline Salviniaceae & 1 & 20.00 & - & - & - & - & 1 & 2.08 \\
\hline Aponogetonaceae & - & - & 1 & 10.00 & - & - & 1 & 2.08 \\
\hline Characeae & - & - & 1 & 10.00 & - & - & 1 & 2.08 \\
\hline Ceratophyllaceae & - & - & 1 & 10.00 & - & - & 1 & 2.08 \\
\hline Convolvulaceae & - & - & 2 & 20.00 & - & - & 2 & 4.16 \\
\hline Marsileaceae & - & - & 1 & 10.00 & - & - & 1 & 2.08 \\
\hline Potamogetonaceae & - & - & 1 & 10.00 & - & - & 1 & 2.08 \\
\hline Amaranthaceae & - & - & - & - & 3 & 9.09 & 3 & 6.25 \\
\hline Asteraceae & - & - & - & - & 5 & 15.15 & 5 & 10.42 \\
\hline Boraginaceae & - & - & - & - & 1 & 3.03 & 1 & 2.08 \\
\hline Commelinaceae & - & - & - & - & 2 & 6.06 & 2 & 4.16 \\
\hline Cyperaceae & - & - & - & - & 5 & 15.15 & 5 & 10.42 \\
\hline Eriocaulaceae & - & - & - & - & 1 & 3.03 & 1 & 2.08 \\
\hline Euphorbiaceae & - & - & - & - & 1 & 3.03 & 1 & 2.08 \\
\hline Fabaceae & - & - & - & - & 2 & 6.06 & 2 & 4.16 \\
\hline Lythraceae & - & - & - & - & 2 & 6.06 & 2 & 4.16 \\
\hline Molluginaceae & - & - & - & - & 1 & 3.03 & 1 & 2.08 \\
\hline Poaceae & - & - & - & - & 3 & 9.09 & 3 & 6.25 \\
\hline Polygonaceae & - & - & - & - & 1 & 3.03 & 1 & 2.08 \\
\hline
\end{tabular}


Checklist Of The Aquatic Macrophyte Flora Abundence In Lower Manair Dam At Karimnagar ....

\begin{tabular}{|l|c|c|c|c|c|c|c|c|}
\hline Scrophulariaceae & - & - & - & - & 4 & 12.12 & 4 & 8.33 \\
\hline Verbenaceae & - & - & - & - & 1 & 3.03 & 1 & 2.08 \\
\hline Typhaceae & - & - & - & - & 1 & 3.03 & 1 & 2.08 \\
\hline & 05 & & 10 & & 33 & & 48 & \\
\hline
\end{tabular}

Table: 8. Number and Percentage contribution of Aquatic weeds to their IUCN Read list (2013.2).

\begin{tabular}{|l|c|c|c|c|c|c|}
\hline \multirow{2}{*}{$\begin{array}{l}\text { IUCN } \\
(2013.2)\end{array}$} & \multicolumn{2}{|c|}{ Free floating } & \multicolumn{2}{c|}{ Submerged weeds } & \multicolumn{2}{c|}{ Emergent weeds } \\
\cline { 2 - 7 } & Total No.s & \% contribution & Total No.s & \% contribution & Total No.s & $\%$ contribution \\
\hline LC & 03 & 6.25 & 08 & 16.67 & 24 & 50.00 \\
\hline NE & 02 & 4.17 & 02 & 4.17 & 08 & 16.67 \\
\hline VU & 0 & 0 & 0 & 0 & 01 & 2.03 \\
\hline
\end{tabular}

Fig: 1. Number of classes, orders, families and species of Macrophytes at lower Manair Dam.

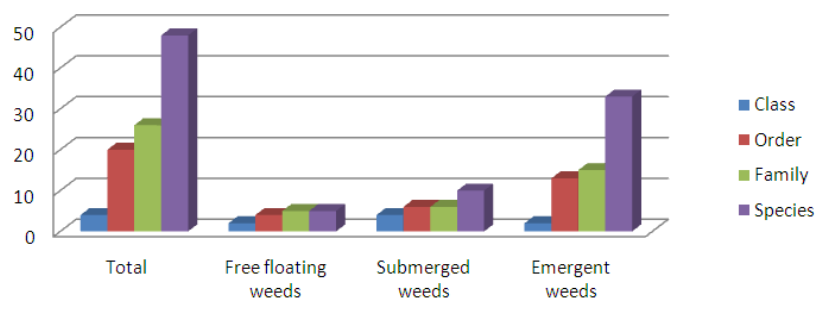

Fig: 2. Number and Percentage contribution of Macrophytes in Four classes.

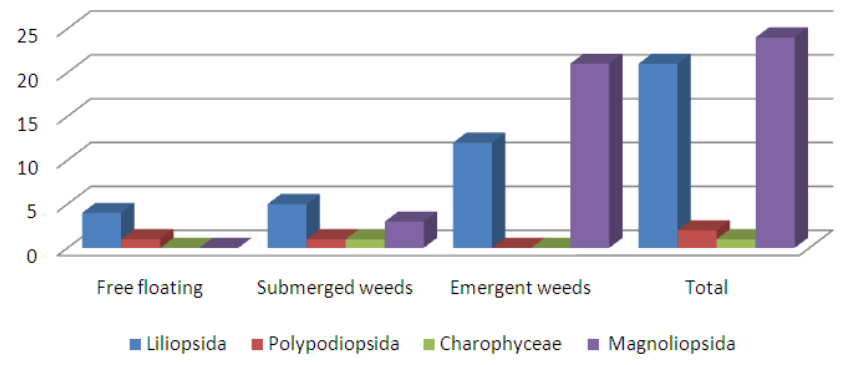

Fig: 3. Number and Percentage contribution of Macrophytes in Twenty orders.

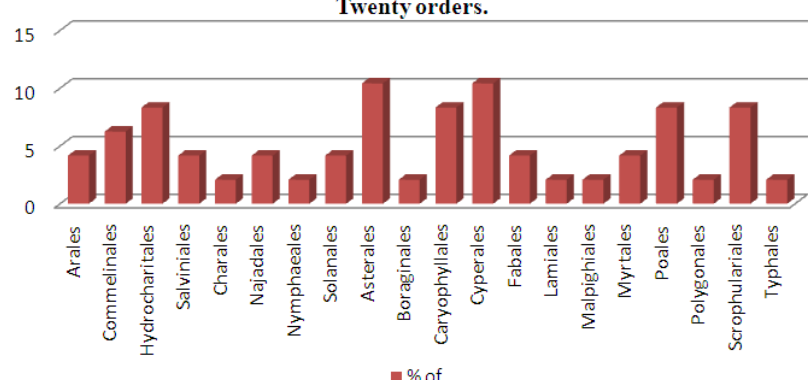

Fig: 4. Number and Percentage contribution of Macrophyte in Twenty six families.
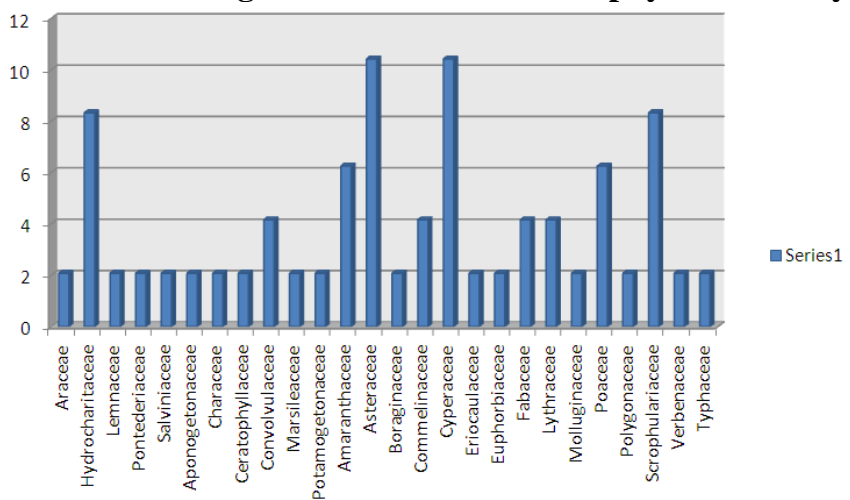
Fig: 5. IUCN Read list 2013.2

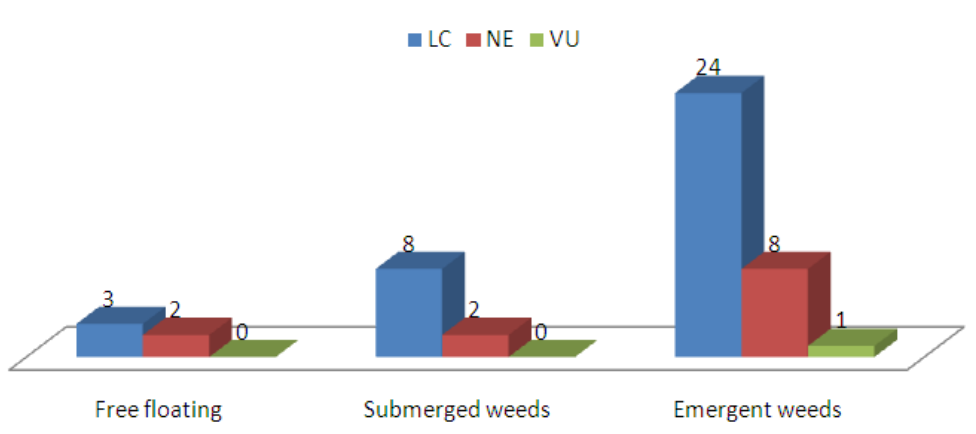

